\title{
Earthquake Probabilities and Energy Characteristics of Seismicity Offshore Southwest Taiwan
}

\author{
Po-Fei Chen ${ }^{1}$, Andrew V. Newman ${ }^{2}$, Tso-Ren $\mathrm{Wu}^{3,{ }^{*}}$, and Ching-Ching $\operatorname{Lin}^{1}$ \\ ${ }^{1}$ Department of Earth Sciences, National Central University, Chung-Li, Taiwan, ROC \\ ${ }^{2}$ Georgia Institute of Technology, Atlanta, Georgia 30332, USA \\ ${ }^{3}$ Institute of Hydrological Sciences, National Central University, Chung-Li, Taiwan, ROC
}

Received 30 November 2007, accepted 26 September 2008

\begin{abstract}
We characterize fault geometries for moderate and large earthquakes in and offshore southwest (SW) Taiwan. Stress orientation estimates suggest that the first shock of the Pingtung earthquake may be characterized by slab flexure. A region of predominately normal-faulting earthquakes is also defined. Using the maximum-likelihood Gutenberg-Richter relation, we find that the repeat times for magnitude, $M \geqq 6.5$ and $M \geqq 7.0$ earthquakes are approximately 30 and 80 years, respectively. By assuming a Gaussian probability distribution with a standard deviation of 0.4 the recurrence time, we estimate a $50 \%$ probability of an $M \geqq 6.5$ earthquake recurring in the next 30 years, while only a $20 \%$ probability of an $M \geqq 7.0$ in the next 50 years. To test the empirical relation that is currently being used to convert $M_{L}$ to seismic moment $\left(M_{0}\right)$, we test the well-defined energy to moment ratio $\left(E / M_{0}\right)$ for the region. We additionally utilize this method to identify the lack of slow-source earthquakes in several recent offshore events in Taiwan.
\end{abstract}

Key words: Taiwan seismicity, Gutenberg-Richter relation, Earthquake probability, Tsunami earthquake

Citation: Chen, P. F., A. V. Newman, T. R. Wu, and C. C. Lin, 2008: Earthquake probabilities and energy characteristics of seismicity offshore southwest Taiwan. Terr. Atmos. Ocean. Sci., 19, 697-703, doi: 10.3319/TAO.2008.19.6.697(PT)

\section{INTRODUCTION}

The region on land and offshore southwestern (SW) Taiwan is considered to be a zone of tectonic escape (Lacombe et al. 2001), and is identified as a zone of reduced seismic activity in Taiwan, as compared to other zones where significant collisional processes are ongoing. However, SW Taiwan is not immune to large earthquakes, as attested by the occurrence of the Pingtung earthquake on 26 December 2006. The two local magnitude $\left(M_{L}\right) 7$ offshore earthquakes (separated by approximately eight minutes) remind us, also of the potential tsunami hazards on SW Taiwan coasts where several devastating events (e.g., Anpin in 1661 and 1782) were documented (Soloviev and Go 1974). Therefore, motivated by the Pingtung earthquake, we analyzed recent activity to estimate recurrence and near future likelihood of an $M_{L} \geqq 7.0$ earthquake in the SW Taiwan region. Likewise, we analyze the energy character of rupture to determine if any of these events have a slow rupture

\footnotetext{
* Corresponding author

E-mail:tsoren@ncu.edu.tw
}

source, an indicative feature of tsunami earthquakes.

While statistical tools, such as frequency-magnitude determinations and probability analysis, are well established, we remark that the results are significantly affected by uncertainties. Hence a careful examination is necessary. Uncertainties arise because of the non-episodic nature of earthquakes, and the short total duration of sampling (e.g., Stein and Newman 2004). For the region, results suggest that the repeat time for an $M_{L} \geqq 7.0$ earthquake is approximately 80 years, with a $20 \%$ probability for another $M_{L} \geqq 7.0$ earthquake occurring within the next 50 years.

For improved tsunami hazard mitigation, a warning system for near-field tsunami detection is currently undertaken. The effectiveness of this system depends heavily on accurate assessment of coastal run-up heights immediately after a tsunamigenic earthquake (Wu et al. 2008). The feasibility of such a system in Taiwan hinges on the "Taiwan Rapid Earthquake Information Release System (TREIRS)" of the Central Weather Bureau (CWB), which generally reports the parameters (longitude, latitude, depth, and $M_{L}$ ) of an earth- 
quake within five minutes of its occurrence. One essential task is to convert the $M_{L}$ scale to seismic moment $\left(M_{0}\right)$, using an empirical rule, for the calculation of approximate seafloor displacements. However, the magnitude to moment transformation may be significantly underestimated for the so-called "tsunami earthquakes" (Kanamori 1972), where the sources rupture in an exceedingly slow fashion. Here, we apply the energy-to-moment ratio discriminate of Newman and Okal (1998) for tsunami earthquake identification for past events offshore Taiwan. We find no energy deficiency that would be characteristic of tsunami earthquakes in the events analyzed. It is thus reasonable to assume that the approximate empirical conversion from the local magnitude to seismic moment is not biased by the occurrence of slow-source tsunami earthquakes.

\section{METHODS}

\subsection{Earthquake Characterization and Tensional Rupture Orientations}

The normal faulting feature of the first shock of the Pingtung earthquake suggests tensional stresses were the dominant controlling mechanism, and is consistent with seismicity associated with tectonic escape in SW Taiwan (Lacombe et al. 2001). In order to constrain the spatial extent of activity, we utilize the distribution of observed seismicity with known focal solutions. We utilized the Global Centroid Moment Tensor (gCMT) catalogue (Dziewonski et al. 1983; www.globalcmt.org), which provides global coverage of earthquake focal mechanisms of moderate and large events (moment magnitude, $M_{W}>5.5$ ) since 1977. Using this cata$\log$ we constrain shallow events (depth $<70 \mathrm{~km}$ ) within an area slightly broader than, and ultimately used to define the study region. A ternary diagram, of the type first used by Frohlich and Apperson (1992), shows the distribution of focal mechanisms within this catalog subset (Fig. 1). From this diagram, it is apparent that activity is predominantly thrust in nature, with about $2 / 3$ 's as many normal faulting events and only a few significant strike-slip events. The Pingtung earthquake is shown to consist first of a strongly normal faulting event, followed by a moderate strike-slip event.

The study region is constrained by the extent of predominant normal faulting activity (Fig. 2; further explained in section 3.2). The northeastern boundary approximately follows the Chishan Transfer Fault Zone, which separates distinctive seismic patterns to the north and south (Lacombe et al. 2001). The west and south boundaries are defined roughly according to the detected range of the CWB TREIRS.

\subsection{Frequency-Magnitude Relationship}

The Gutenberg and Richter (GR) law describes that the number of earthquakes $(N)$ with magnitude larger than or equal to $M$ follows a power law distribution (Gutenberg and
Richter 1944), such that:

$\log _{10} N(M)=a-b M$

the $b$-value indicates the relative size and frequency of occurrence of earthquakes. Since the CWB catalogue is the source of seismicity and it reports local magnitude $\left(M_{L}\right)$, we thus use $M_{L}$ for the GR law analysis. The $M_{L}$ of earthquakes in the CWB catalogue are routinely determined following the formula proposed by Richter (1935). The peak amplitude is measured on simulated Wood-Anderson seismograms, which are synthesized by firstly deconvolving the known instrument response and then convolving the response of the standard Wood-Anderson seismometer (natural period at $0.8 \mathrm{sec}$ ). An empirical law suitable for the Taiwan region is adopted for correcting the effects of hypocentral distances (Shin 1993).

We retain only shallow earthquakes $(0-70 \mathrm{~km})$ within the defined study region for the determination of the GR

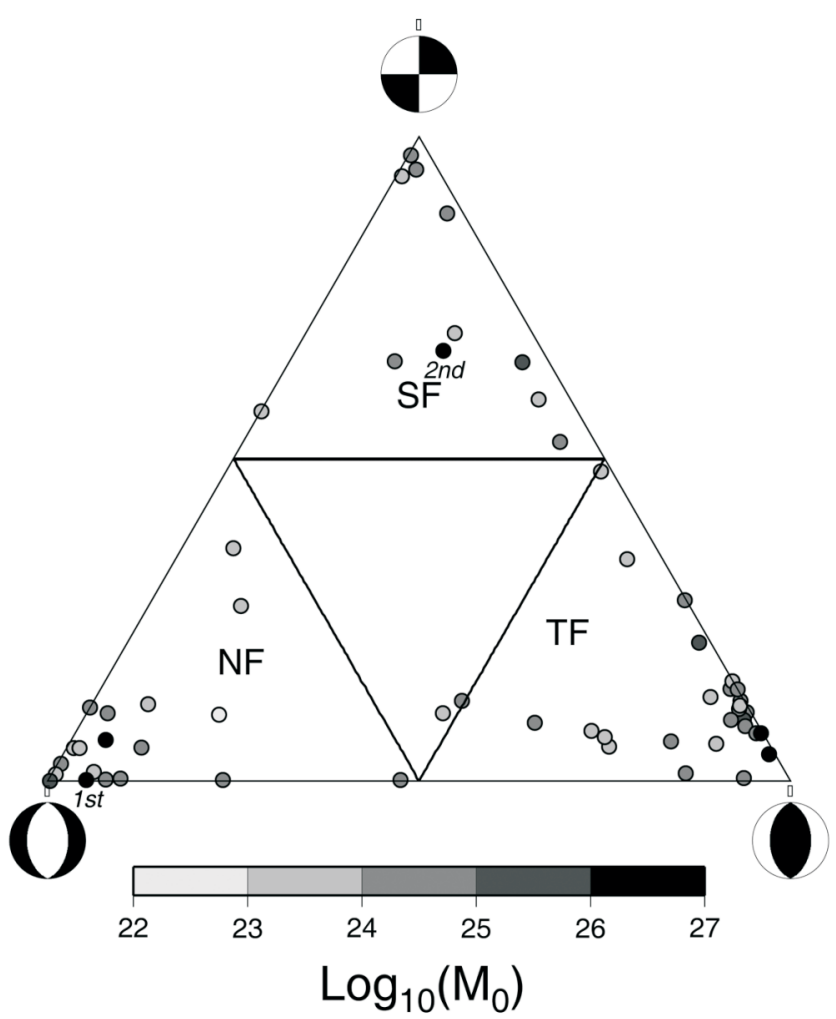

Fig. 1. Ternary diagram displaying the distribution of earthquake focal mechanisms. The three apexes indicate a pure strike-slip (top), a pure normal fault with $45^{\circ}$ dipping plane (left), and a pure thrust fault with $45^{\circ}$ dipping plane (right). Fractions of the three components for sampled earthquakes are indicated by their positions inside the triangle. The three solid lines are the boundaries to define the strike-slip fault group (SF), the normal fault group (NF), and the thrust fault group (TF), respectively. The seismic moment of individual events are scaled by increased shading. The closer an earthquake projects to a corner, the more dominant that rupture type is. Results of the Pingtung doublet earthquakes are indicated as $1^{\text {st }}$ and $2^{\text {nd }}$, respectively. 


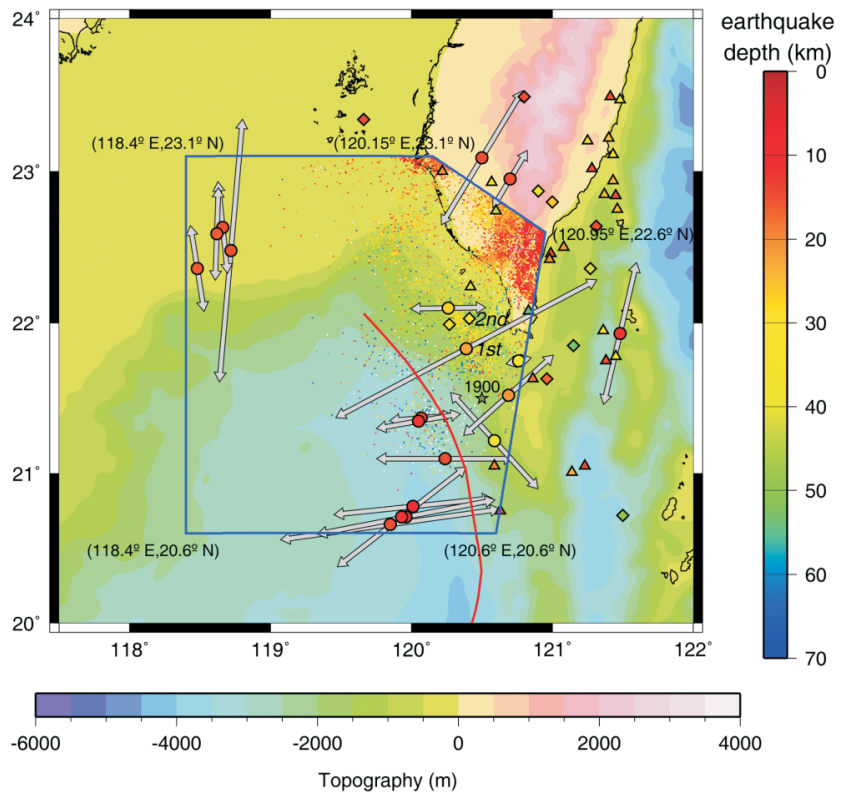

Fig. 2. Spatial distributions of the gCMT events and normal faulting tensional axes. Normal (circles), thrust (triangles), and strike-slip (diamonds) events are identified and color-coded with depth. The horizontal projections of tensional axes for normal faulting earthquakes are shown with length proportional to event size. Area within the blue box is the study region constrained by the occurrence of predominantly normal faulting earthquakes, and extending to the detectable range of the CWB catalogue (yellow dots). Red line indicates the Manila trench.

relation. Upon investigating the cumulative number of events as a function of year, we find that the slope changes from nearly flat to relatively steep by 1979 , corresponding to the installation of the Taiwan Telemetered Seismographic Network between 1972 and 1987 (Wang 1989). We thus deem that the CWB catalogue is nearly complete beginning in 1979 and only the time period between 1979 and 2007 is analyzed.

The maximum-likelihood technique for calculating the $b$-value is adopted (Aki 1965; Woessner and Wiemer 2005):

$b=\frac{\log _{10}(e)}{\left[\langle M\rangle-\left(M_{c}-\frac{\Delta M_{b i n}}{2}\right)\right]}$

where $e$ is approximately $2.718,\langle M\rangle$ is the mean magnitude, $M_{c}$ is the minimum magnitude of completeness defined as the lowest magnitude at which $100 \%$ of the events are detected in the catalog, and $\Delta M_{\text {bin }}$ is the binning width (0.01 in this study). To determine the optimal $M_{c}$ of the catalog, approaches of Wiemer and Wyss (2000) are followed. Firstly, the $a$ - and $b$-value as a function of minimum magnitudes $\left(M_{i}\right)$ and an infinite maximum magnitude are determined. Secondly, residuals $(R)$ for each derivation are calculated using (Wiemer and Wyss 2000):

$R\left(a, b, M_{i}\right)=\frac{\sum_{M_{i}}^{M_{\max }}\left|B_{i}-S_{i}\right|}{\sum_{i} B_{i}} \times 100$

where $B_{i}$ and $S_{i}$ are the cumulative number of events in each magnitude bin from observation and synthetics. $M_{\max }$ is the magnitude of largest earthquakes in the dataset. The optimal $M_{c}$ is then determined at which $R$ is minimum (Fig. 3). Furthermore, to alleviate the saturation problem of $M_{L}$ scale, we set $M_{L}=6.0$ as the upper bound magnitude for the determination of $a$ - and $b$-values.

\subsection{Probability Analysis}

From the derived GR law, we extrapolate to larger events at lower recurrence rates to estimate the repeat time of earthquakes with a given magnitude $(M)$. Because $M_{L}$ begins to saturate near $M_{L}=6.5$, where rupture durations significantly exceed the natural period of the Wood-Anderson seismometer it is necessary to continue with a generic magnitude characterization, $M$. This type of relationship has been shown using body-wave magnitudes (Okal and Romanowicz 1994), and its application is further described in Newman et al. (1999). We estimate the probabilities of two scenarios

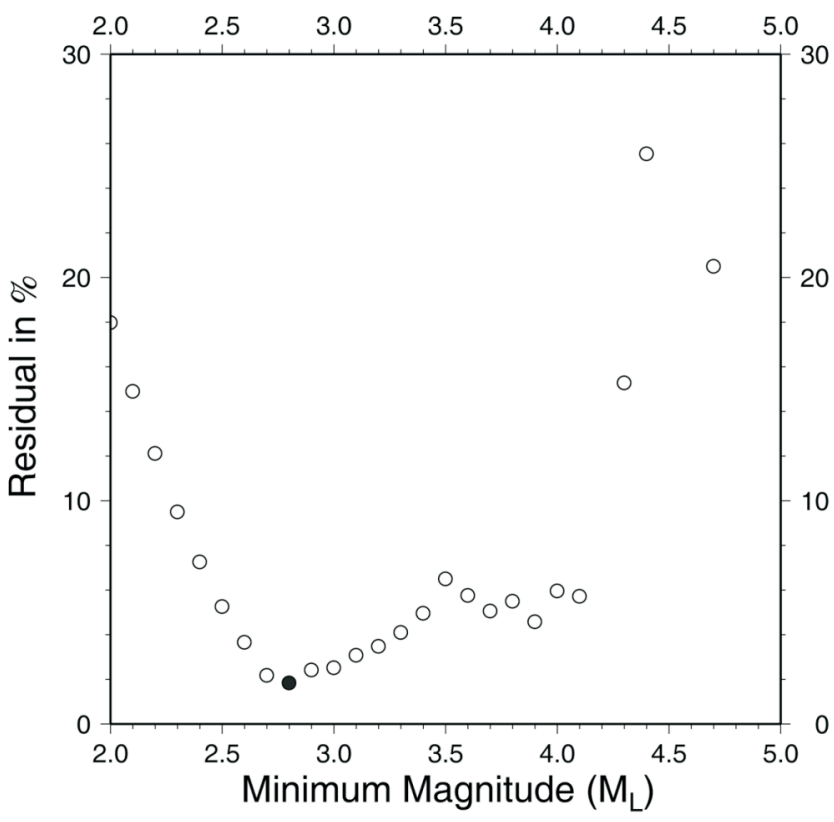

Fig. 3. Minimum magnitude of completeness, $M_{c}$, is shown as a function of residual to the Gutenberg-Richter (GR) determination. The optimal $M_{c}$ is 2.8 (solid circle), and is determined to be the magnitude cut-off with the lowest overall residual. The ultimate GR relation for this study is highly sensitive to this value, and hence an accurate determination is necessary. 
within the study region: (1) an $M \geqq 6.5$ earthquake to occur in the next 30 years, and (2) an $M \geqq 7.0$ earthquake to occur in the next 50 years. The estimation is equivalent to calculating the conditional probability of an earthquake occurring between $\mathrm{T}_{0}$ (the year 2007) and $\mathrm{T}$ (the year 2037 for an $M \geqq$ 6.5 and the year 2057 for an $M \geqq 7.0$ ). The probability density function is assumed to be a Gaussian distribution with the mean taken as the repeat time, and the standard deviation taken as 0.4 of the mean repeat time (Nishenko and Buland 1987).

\subsection{The $E / M_{0}$ Discriminant for Tsunami Earthquakes}

We adopted the dimensionless parameter $\Theta=\log _{10}$ $\left(E / M_{0}\right)$ as proposed by Newman and Okal (1998) to discriminate tsunami earthquake from recent large offshore earthquakes around Taiwan (not limited to the study area). A tsunami earthquake will exhibit a reduction of one or more units in $\Theta$, representing at least a 10 -fold decrease in radiated energy $(E)$ than expected for its seismic moment $\left(M_{0}\right)$. This is because tsunami earthquakes rupture more slowly, having a low corner frequency, and hence have minimal shaking for the same amount of slip. While $M_{0}$ can readily be obtained from the gCMT catalogue, we calculate the energy using the algorithm given by Boatwright and Choy (1986) and modified by Newman and Okal (1998).

Briefly, the energy algorithm calculates the energy flux of the generalized $\mathrm{P}$ wave group $(P, p P$, and $s P)$ recorded at teleseismic stations, which has been corrected both for instrument response and receiver function (Okal 1992). The calculated flux is then scaled back to a focal sphere, accounting for radiation pattern, geometrical spreading and frequency-dependent attenuation (Newman and Okal 1998). The radiated seismic energy is finally obtained by integrating over the unit sphere, and adding those of the generalized $\mathrm{S}$ wave group $(S, p S$, and $s S)$, expressed as a constant correction for the ratio of Energy in the P vs. S waves of 4/15 (Boatwright and Fletcher 1984).

\section{RESULTS}

\subsection{Earthquake Characterization and Tensional Rupture Orientations}

Figure 1 indicates that the first shock of the Pingtung earthquake is normal faulting, whereas the second shock strike-slip faulting. We group the results of earthquakes in the gCMT catalogue into three categories (Fig. 1): normal faulting (NF), strike slip faulting (SF), and thrust faulting (TF). Of the 57 earthquakes in the catalog (excluding the Pingtung doublet), approximately $42 \%$ of activity is strongly thrust in nature, while about $26 \%$ is strongly normal faulting, and only $7 \%$ is strongly strike-slip. The rest of the seismicity is a mixture of these mechanisms, but are less dominant in orientation, yielding inferred stress orientations without a near-vertical principle component.

The orientations of tension axes from normal faulting earthquakes are examined geospatially (Fig. 2). The two on-land events in SW Taiwan show NE-SW trending tension axes, consistent with tectonic escape. However the four events in the Taiwan strait exhibit strong N-S trending axes suggesting a dominant and presumably unassociated extension. Most interestingly, almost all events south of Taiwan (including the Pingtung first shock) have predominantly E-W tension axes. These axes are near-normal to the trench, suggesting their origins is due to bending stresses in the slab.

\subsection{Spatial Patterns of Different Earthquake Rupture Types and Determination of Study Area}

A spatial pattern is observed that while most normalfaulting earthquakes, possibly caused by tectonic escape or slab bending, are located to the south and west of southern Taiwan, most thrust-faulting earthquakes, occurring as a result of Philippine-Eurasia collision, occur in a linear N-S trend to the east (Fig. 2). This pattern is consistent with the geological understanding of the tectonic setting of the Hengchun Peninsula. It is the spatial pattern that enables us to define the boundary of the study region consisting of mainly normal faulting earthquakes in and to the southwest of Taiwan and extending the approximate coverage range of the CWB TREIRS (Fig. 2).

\subsection{Frequency-Magnitude Analysis}

Residuals as a function of minimum magnitude used show that the optimal value occurs at $M_{L}=2.8$ (Fig. 3). We thus use earthquakes with $2.8 \leqq M_{L} \leqq 6.0$ to determine the GR law for the study region. The best fitting GR relation is determined to be $\log _{10} N=5.92-0.87 M_{L}$ (Fig. 4). We use this result to extrapolate the recurrence times for larger $M$ 6.5 and 7.0 at approximately 30 and 80 years, respectively. Because earthquakes larger than this size will likely have elongated rupture dimensions due to finiteness of rupture depth, great caution should be used in further extrapolation to even larger magnitudes. Any such direct extrapolation will likely underestimate recurrence times.

The results from extrapolation are roughly consistent with the yearly moment release of earthquakes for the past century within the study region (Fig. 5), where $M \geqq 7.0$ events only occurred in 1900 and 2006. Figure 5 is produced by the conversion relation $\log _{10}\left(M_{0}\right)=1.45 M_{L}+16.03$, based on a linear fitting of common earthquakes recorded by the CWB catalogue and the gCMT catalogue within the study region.

\subsection{Earthquake Probabilities}

Given the 30 - and 80-year repeat times, we construct the 
Frequency-Magnitude Relationship (1979-2007)

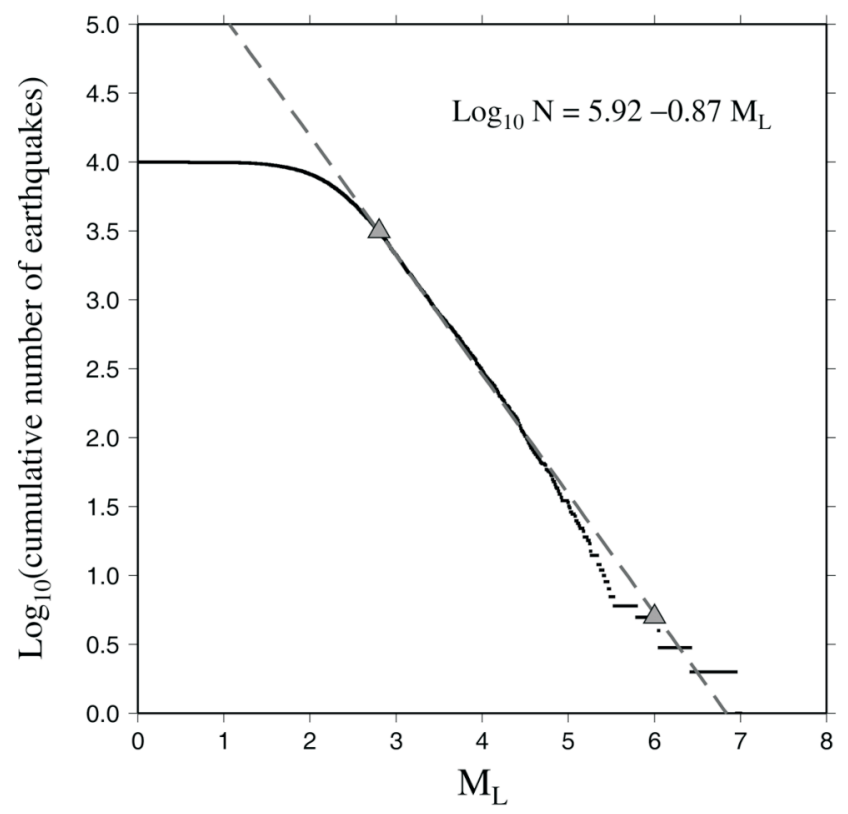

Fig. 4. The GR relation (grey dashed line), as determined by the maximum likelihood method, is determined using $M_{c}$ (upper-left triangle). Dots represent the observed earthquake populations binned at $0.01 M_{L}$. The upper magnitude bound (lower-right triangle) is chosen at a point before $M_{L}$ begins to saturate (6.0).

\section{Yearly moment release}

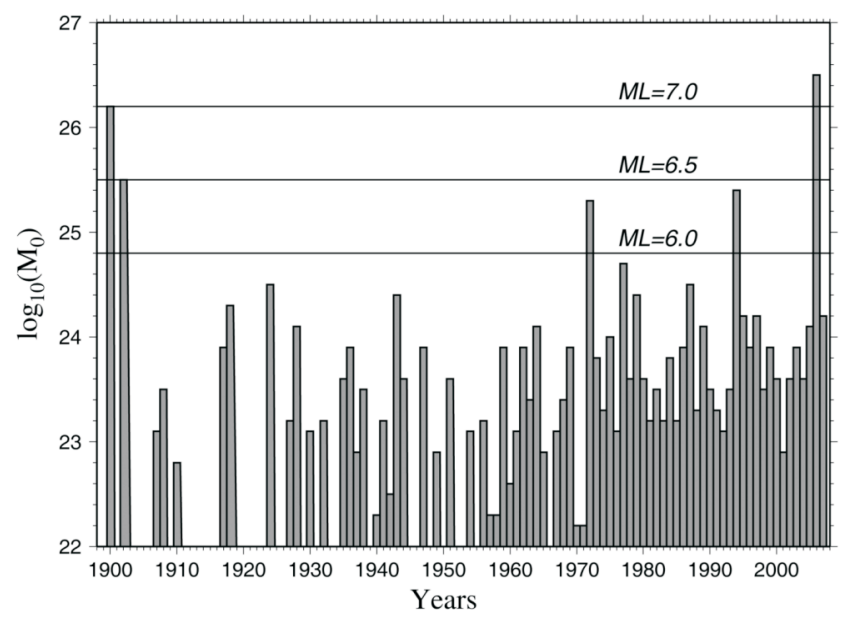

Fig. 5. The annual moment release for the study area is shown for events within the CWB catalogue. Horizontal lines represent the regionally determined $M_{L}$ equivalent. An empirical relation describes the conversion from the regional $M_{L}$ to seismic moments, $M_{0}$ : $\log _{10}\left(M_{0}\right)=$ $1.45 M_{L}+16.03$. In only two years (1900 and 2006), have the cumulative annual release exceeded the $M_{L}=7.0$ line.

Gaussian distribution, with 12- and 32-year standard deviation, of the probability density distribution relative to the year 2007, corresponding to the Dec. 2006 Pingtung earthquake (top Fig. 6). Upon calculations of the conditional probabilities, we estimate that the probability of an $M \geqq 6.5$ earthquake to occur in the next 30 years is about $50 \%$ and the probability of an $M \geqq 7.0$ earthquake to occur in the next 50 years is about $20 \%$ (bottom Fig. 6). As shown in Fig. 6 , the 30 - or 50 -year probability will increase with the reference year.

\subsection{The E/M $/ \mathbf{M}_{0}$ Discriminant for Tsunami Earthquakes}

We analyzed available teleseismic data of the 20 Sep. 1999 Chi-Chi earthquake (out of curiosity), along with the 31 Mar. 2002 Hualein earthquake, the 10 Dec. 2003 Chengkung earthquake, and the first shock of the 26 Dec. 2006 Pingtung earthquake. None exhibit reduced energy radiation, having $\bar{\Theta} \sim-4.5$, and hence do not exhibit features indicative of tsunami earthquakes (Fig. 7). The near constant $\Theta$ value suggests all events have similar rupture speeds and dimensions. While none of these earthquakes exhibited a tsunami-earthquake component, this does not preclude the possibility of future such events occurring in this region. This is evident in the study of Okal and Newman (2001), where other moderate and large earthquakes in the zones of previous tsunami earthquakes were analyzed, finding that no such tsunami earthquake character were present in most events.

\section{DISCUSSION}

The rupture processes of the Dec. 2006 Pingtung earthquake doublet have been inverted by various methods to understand the kinetic behavior (e.g., Huang et al. 2008; Lee et al. 2008; Yen et al. 2008). Dynamically, the orientation of tension axis of the first shock exhibits a direction roughly normal to the strike of the Manila trench, suggesting that the causing stresses may be associated with slab flexure, bending or unbending, with the latter proposed by Liao et al. (2008). In any case, it justifies the presence of the Luzon slab here, while the surface feature of the Manila trench is rather obscure.

The tensional-stress environment in the SW Taiwan onland and offshore, as demonstrated by the spatial pattern of the gCMT focal solutions enables us to isolate seismicity of one type for statistical analysis. Although the $b$-value of normal faulting events is expected to be higher than those of thrust or strike-slip events (Schorlemmer et al. 2005), the obtained $b=0.87$ is less than the universal observed constant of $b \sim 1.0$. This may represent characteristics of the study area and/or the CWB catalogue. Furthermore, although the south and west boundaries of the box to group the study area are constrained by the detectable range of the CWB catalogue, it is obvious that areas relatively far away from the main Taiwan island suffers problems of undersampling as shown by the sparse seismicity. As a result, the probability for an $M \geqq 7.0$ earthquake to occur within the study area would be somewhat higher than $20 \%$. 

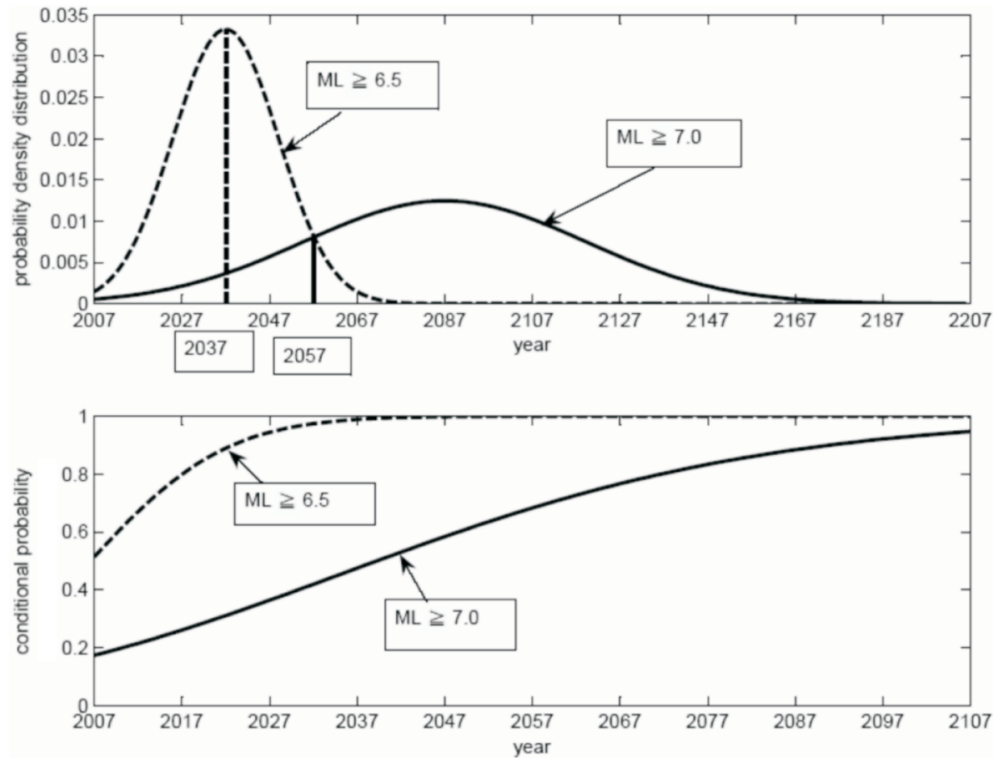

Fig. 6. Earthquake probabilities for the study area estimated for large earthquakes beginning in 2007 ( 1 year after the last major event). Top: The assumed Gaussian distribution of probability density functions are shown. Solid and dashed lines indicate the probability distribution of an $M \geqq 7.0$, and $M \geqq 6.5$ earthquake, respectively. Vertical lines indicate the time intervals for estimating the conditional probability for each case. Bottom: Conditional probabilities show approximately $50 \%$ chance of an $M \geqq 6.5$ event occurring in the next 30 years (dashed line), and almost a $20 \%$ chance an $M \geqq 7.0$ earthquake occurring in the next 50 years (solid line). Note that generic magnitudes $(M)$ are used here because $M_{L}$ suffers significantly from saturation in this range.

\section{CONCLUSIONS}

We observe a spatial pattern of moderate and large earthquakes with different rupture mechanisms; while earthquakes on and offshore SW Taiwan, and below the Manila accretionary wedge exhibit normal faulting, possibly caused by tectonic escape or slab bending, earthquakes to the right (on the arc) display predominantly thrust faulting, due to Philippine-Eurasia collision. The Gutenberg-Richter powerlaw distribution of seismicity within the tensionally-stressed area suggests that earthquakes of $M \geqq 7.0$ event will recur about once every 80 years. After the 26 Dec. 2006 Pingtung doublet earthquakes, we estimate that the probability of an $M \geqq 7.0$ earthquake to occur in the area within the next 50 years is about $20 \%$. We calculated the $E / M_{0}$ ratio of past four Taiwanese earthquakes: the $20 \mathrm{Sep} .1999$ Chi-Chi earthquake, the 31 Mar. 2002 Hualein earthquake, the 10 Dec. 2003 Chengkung earthquake and the 26 Dec. 2006 Pingtung earthquake (the first shock). Results suggest that none exhibit deficient energy, and hence none have features of tsunami earthquake rupture. Because the $E / M_{0}$ ratios for these four events are near constant, it is reasonable to assume that the approximate empirical conversion from our local mag-

$E / M_{0}$ for large Global Earthquakes

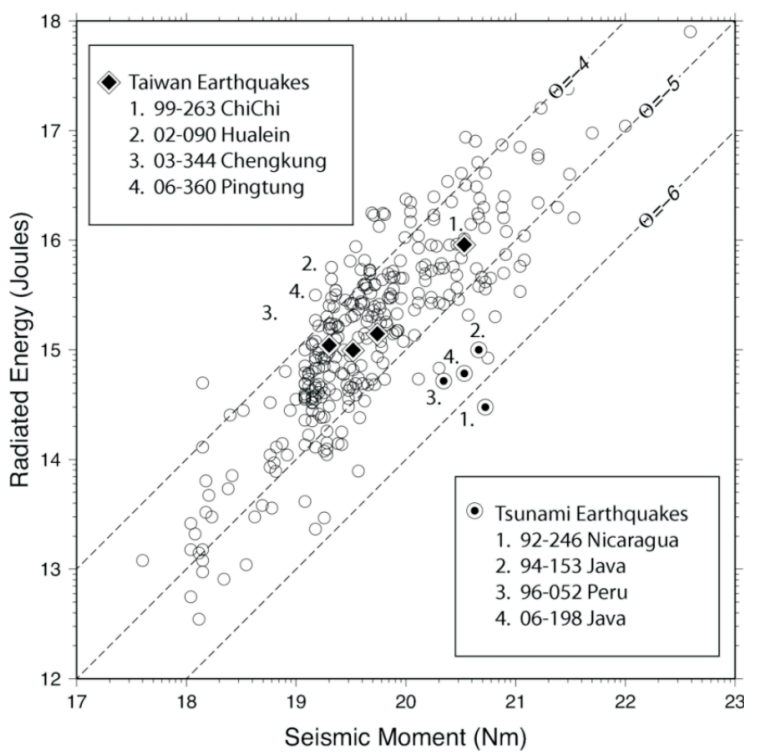

Fig. 7. Energy-Moment relationship for the global distribution of seismicity (Newman and Convers 2008) along with the ChiChi and regional offshore earthquakes near SW Taiwan (filled diamonds). Diagonal lines correspond to constant values of $\Theta=\log _{10}\left(E / M_{0}\right)$. Tsunami earthquakes (filled circles) are identified by a deficiency of nearly one unit of magnitude or more in $\Theta$ (Newman and Okal 1998). It is evident here that the events identified in SW Taiwan, and including the ChiChi earthquake have a near constant $\Theta \sim-4.5$, nowhere near the tsunami earthquake threshold of $\Theta<-5.7$. nitude to seismic moment is not biased by tsunami earthquakes. However, caution should be taken, because this does not preclude the likelihood of a tsunami earthquake occurring in this region in the future. If so, that event will yield an underestimate of the seismic moment by as much as a factor of 10 or more.

Acknowledgements We thank Central Weather Bureau in Taipei for earthquake catalogue. We thank W. H. K. Lee and E. A. Okal for improvements of the manuscript. This research was supported by the Taiwan Earthquake Research Center (TEC) funded through National Science Council (NSC) with grant number 95-2116-M-008-002. The TEC contribution number for this article is 00039 .

\section{REFERENCES}

Aki, K., 1965: Maximum likelihood estimate of $b$ in the formula $\log N=a-b M$ and its confidence limits. Bull. Earthq. Res. Inst., Tokyo Univ., 43, 237-239.

Boatwright, J. and J. B. Fletcher, 1984: The partition of radiated energy between $\mathrm{P}$ and S waves. Bull. Seismol. Soc. Am., 74, 361-376.

Boatwright, J. and G. L. Choy, 1986: Teleseismic estimates of the energy radiated by shallow earthquakes. J. Geophys. Res., 91, 2095-2112. 
Dziewonski, A. M., A. Friedman, D. Giardini, and J. H. Woodhouse, 1983: Global seismicity of 1982: Centroid-momenttensor solutions for 308 earthquakes. Phys. Earth Planet. Inter., 33, 76-90.

Frohlich, C. and K. D. Apperson, 1992: Earthquake focal mechanisms, moment tensors, and the consistency of seismic activity near plate boundaries. Tectonics, 11, 279-296.

Gutenberg, R., and C. F. Richter, 1944. Frequency of earthquakes in California. Bull. Seismol. Soc. Am., 34, 185-188.

Huang, B. S., Y. L. Huang, S. J. Lee, Y. G. Chen, and J. S. Jiang, 2008: Initial rupture processes of the 2006 Pingtung earthquake from near source strong-motion records. Terr. Atmos. Ocean. Sci., 19, 547-554, doi: 10.3319/TAO.2008. 19.6.547(PT).

Kanamori, H., 1972: Mechanism of tsunami earthquakes. Phys. Earth Planet. Inter., 6, 346-359.

Lacombe, O., F. Mouthereau, J. Angelier, and B. Deffontaines, 2001: Structural geodetic and seismological evidence for tectonic escape in SW Taiwan. Tectonophysics, 333, 323345.

Lee, S. J., W. T. Liang, and B. S. Huang, 2008: Source mechanisms and rupture processes of the 26 December 2006 Pingtung earthquake doublet as determined from the regional seismic records. Terr. Atmos. Ocean. Sci., 19, 555-565, doi: 10.3319/TAO.2008.19.6.555(PT).

Liao, Y. C., S. K. Hsu, C. H. Chang, W. B. Doo, M. Y. Ho, C. L. Lo, and C. S. Lee, 2008: Seismic tomography off SW Taiwan: A joint inversion from OBS and onshore data of 2006 Pingtung aftershocks. Terr. Atmos. Ocean. Sci., 19, 729-741, doi: 10.3319/TAO.2008.19.6.729(PT).

Newman, A. V. and E. A. Okal, 1998: Teleseismic estimates of radiated seismic energy: The $\mathrm{E} / \mathrm{M}_{0}$ discriminant for tsunami earthquakes. J. Geophys. Res., 103, 26885-26898.

Newman, A. V., S. Stein, J. C. Weber, J. F. Engeln, A. Mao, and T. H. Dixon, 1999: Slow deformation and implied long earthquake recurrence intervals from GPS surveys across the New Madrid seismic zone. Science, 284, 619-621.

Newman, A. V. and J. A. Convers, 2008: A rapid high-frequency energy-duration discriminant for very large and tsunami earthquakes. Geoph. Res. Lett., in review.

Nishenko, S. P. and R. Buland, 1987: A generic recurrence interval distribution for earthquake forecasting. Bull. Seismol. Soc. Am., 77, 1382-1399.
Okal, E. A., 1992: A student's guide to teleseismic body-wave amplitudes. Seismol. Res. Lett., 63, 169-180.

Okal, E. A. and B. A. Romanowicz, 1994: On the variation of b-value with earthquake size. Phys. Earth Planet. Inter., 87, 55-76.

Okal, E. A. and A. V. Newman, 2001: Tsunami earthquakes: The quest for a regional signal. Phys. Earth Planet. Inter., 124, 45-70.

Richter, C. F., 1935: An instrumental magnitude scale. Bull. Seismol. Soc. Am., 25, 1-32.

Schorlemmer, D., S. Wiemer, and M. Wyss, 2005: Variations in earthquake-size distribution across different stress regimes. Nature, 437, 539-542.

Shin, T. C., 1993: The calculation of local magnitude from the simulated Wood-Anderson seismograms of the short-period seismograms in the Taiwan area. Terr. Atmos. Ocean. Sci., 4, 155-170.

Soloviev, S. L. and Ch. N. Go, 1974: A catalogue of tsunamis on the western shore of the Pacific Ocean (173-1968). Nauka Publishing House, Moscow, USSR, 310 pp, Can. Transl. Fish. Aquat. Sci. 5077, 1984.

Stein, S. and A. V. Newman, 2004: Characteristic, uncharacteristic, and absent earthquakes as possible artifacts of short earthquake histories. Seismol. Res. Lett., 75, 173-187.

Wang, J. H., 1989: The Taiwan telemetered seismographic network. Phys. Earth Planet. Inter., 58, 9-18.

Wiemer, S. and M. Wyss, 2000: Minimum magnitude of complete reporting in earthquake catalogs: Examples from Alaska, the Western United States, and Japan. Bull. Seismol. Soc. Am., 90, 859-869.

Woessner, J. and S. Wiemer, 2005: Assessing the quality of earthquake catalogues: Estimating the magnitude of completeness and its uncertainty. Bull. Seismol. Soc. Am., 95, 684-698, doi: 10.1785/0120040007.

Wu, T. R., P. F. Chen, W. T. Tsai, and G. Y. Chen, 2008: Numerical study on tsunamis excited by 2006 Pingtung earthquake doublet. Terr. Atmos. Ocean. Sci., 19, 705-715, doi: 10.3319/TAO.2008.19.6.705(PT).

Yen, Y. T., K. F. Ma, and Y. Y. Wen, 2008: Slip partition of the 26 December 2006 Pingtung, Taiwan (M 6.9, M 6.8) earthquake doublet determined from teleseismic waveforms. Terr. Atmos. Ocean. Sci., 19, 567-578, doi: 10.3319/TAO. 2008.19.6.567(PT). 